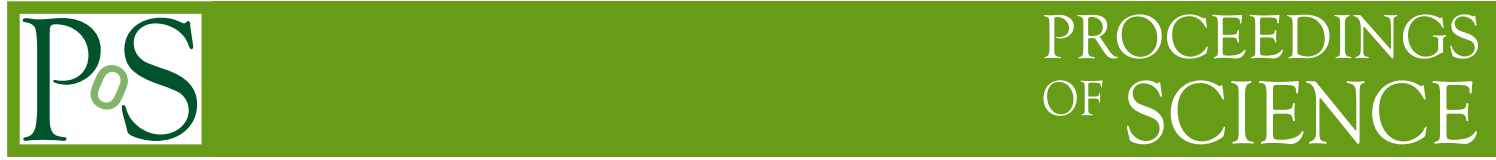

\title{
KTeV Results on Kaon Radiative Decays
}

\section{E. Cheu*}

University of Arizona, Tucson, AZ, USA

E-mail: elliott@physics.arizona.edu

The $\mathrm{KTeV}$ experiment has carried out a broad program of studies of rare kaon decays. In this paper we present results on $K_{L} \rightarrow \pi^{0} \gamma \gamma, K_{L} \rightarrow \pi^{0} e^{+} e^{-} \gamma$ and $K_{L} \rightarrow \pi^{ \pm} e^{\mp} v e^{+} e^{-}$. These decays provide a window for testing chiral perturbation theory. We find $\operatorname{BR}\left(K_{L} \rightarrow \pi^{0} \gamma \gamma\right)=(1.30 \pm$ $0.03) \times 10^{-6}, \mathrm{BR}\left(K_{L} \rightarrow \pi^{0} e^{+} e^{-} \gamma\right)=(1.90 \pm 0.16 \pm 0.12) \times 10^{-8}$, and $\mathrm{BR}\left(K_{L} \rightarrow \pi^{ \pm} e^{\mp} v e^{+} e^{-}\right)$ $=(1.29 \pm 0.01 \pm 0.04) \times 10^{-5}$. The $\mathrm{KTeV}$ measurements are competitive with or better than the world's best results in these decays.

KAON International Conference

May 21-25 2007

Laboratori Nazionali di Frascati dell'INFN, Rome, Italy

\footnotetext{
${ }^{*}$ Speaker.
} 
The decays $K_{L} \rightarrow \pi^{0} \gamma \gamma, K_{L} \rightarrow \pi^{0} e^{+} e^{-} \gamma$ and $K_{L} \rightarrow \pi^{ \pm} e^{\mp} v e^{+} e^{-}$can all be used as tests of chiral perturbation theory. In the case of the first two modes, the predictions for the branching ratios of these modes show significant increases when one uses $O\left(p^{6}\right)$ versus $O\left(p^{4}\right)$ chiral perturbation theory. The first measurements of $K_{L} \rightarrow \pi^{0} \gamma \gamma[1,2]$ were a factor of three higher than the $O\left(p^{4}\right)$ prediction, but were consistent with the $O\left(p^{6}\right)$ calculation [3]. This was also seen in the decay $K_{L} \rightarrow \pi^{0} e^{+} e^{-} \gamma$ where the $O\left(p^{4}\right)$ prediction was inconsistent with the measurement, but consistent with the $O\left(p^{6}\right)$ calculation [4]. For the $K_{L} \rightarrow \pi^{ \pm} e^{\mp} v e^{+} e^{-}$mode, the model of the $K-\pi$ current can be used as a probe of chiral perturbation theory [5].

In addition, the two decays $K_{L} \rightarrow \pi^{0} \gamma \gamma$ and $K_{L} \rightarrow \pi^{0} e^{+} e^{-} \gamma$ are important for understanding the direct CP violating decay $K_{L} \rightarrow \pi^{0} e^{+} e^{-}$. Three components contribute to the $K_{L} \rightarrow \pi^{0} e^{+} e^{-}$ amplitude: direct $\mathrm{CP}$ violation, indirect $\mathrm{CP}$ violation (and an interference term), and a $\mathrm{CP}$ conserving term. Recent measurements of the decay $K_{S} \rightarrow \pi^{0} e^{+} e^{-}[6]$ and $K_{S} \rightarrow \pi^{0} \mu^{+} \mu^{-}$[7] have helped to determine the indirect CP violating contributions to $K_{L} \rightarrow \pi^{0} e^{+} e^{-}$and $K_{L} \rightarrow \pi^{0} \mu^{+} \mu^{-}$. The magnitude of the $\mathrm{CP}$ conserving contributions to $K_{L} \rightarrow \pi^{0} l^{+} l^{-}$can be determined by measurements of the decay $K_{L} \rightarrow \pi^{0} \gamma \gamma[8,9]$ and $K_{L} \rightarrow \pi^{0} e^{+} e^{-} \gamma$. The CP conserving term is estimated to be small[10].

\section{The KTeV Experiment}

The KTeV experiment is located at Fermilab. The detector contains a charged spectrometer with four drift chambers, two on either side of a large dipole magnet. At the downstream end of the detector is a $1.9 \mathrm{~m} \times 1.9 \mathrm{~m}$ calorimeter consisting of 3100 pure CsI blocks. Following the calorimeter are $10 \mathrm{~cm}$ of lead and 5 meters of steel which act as a muon filter. Two planes of scintillator, used for muon detection, are located just downstream of the steel. Photon vetoes to detect the presence of particles that would otherwise escape detection surround the spectrometer. The charged spectrometer achieves a hit resolution of better than $100 \mu \mathrm{m}$, while the CsI calorimeter obtains better than $1 \%$ energy resolution over the range of energies of interest. A transition radiation detector (TRD) capable of $e / \pi$ separation of 200:1 with $90 \%$ efficiency is located just upstream of the CsI calorimeter.

The $\mathrm{KTeV}$ experiment utilized two configurations and took data during a number of different periods between 1996 and 1999. In the E832 configuration, we had three different running periods: 1996, 1997 and 1999. The E799 running occurred in 1997 and 1999. For the entire E799 data set, approximately $6.6 \times 10^{11}$ kaon decays occurred in the $\mathrm{KTeV}$ detector. This large kaon flux allows us to have an unprecedented sensitivity to a number of rare kaon decays with large multiplicity final states.

\section{Measurement of $K_{L} \rightarrow \pi^{0} \gamma \gamma$}

In the decay $K_{L} \rightarrow \pi^{0} \gamma \gamma$, the final state consists of four photons, two from the $\pi^{0}$ and two from the $K_{L}$. Our analysis requires four electromagnetic clusters in the CsI calorimeter, with no tracks or extra clusters. Of the six possible combinations of two-photon pairs, we choose the combination with the two photon invariant mass closest to the $\pi^{0}$ mass. 
Backgrounds to this decay originate from two main sources, $K_{L} \rightarrow \pi^{0} \pi^{0}$ and $K_{L} \rightarrow \pi^{0} \pi^{0} \pi^{0}$. The first background has the same topology as our signal events. However, the relatively small branching ratio for $K_{L} \rightarrow \pi^{0} \pi^{0}$ and kinematic cuts reduce this background to a negligible level. In particular, we look at all possible photon combinations of the four photons and reject any event in which the invariant $\gamma \gamma$ mass for both pairs of photons is reconstructed near the $\pi^{0}$ mass.

$K_{L} \rightarrow \pi^{0} \pi^{0} \pi^{0}$ decays constitute the largest source of background to $K_{L} \rightarrow \pi^{0} \gamma \gamma$. These events can contribute to the background when photons either miss the calorimeter or two or more photons overlap in the CsI calorimeter. These backgrounds can be reduced by eliminating events with signals in the photon vetoes. In addition, one can improve the signal to background ratio by cutting on the reconstructed $z$ position of the event because events with missing photons reconstruct downstream of the true decay position.

To get rid of events in which photons overlap in the CsI calorimeter, we define a photon shape variable. This variable uses the $3 \times 3$ array of CsI crystals containing the core of the shower and compares the energy distribution to an ideal energy distribution determined from Monte Carlo. Large values of this shape variable are indicative of overlapping photons.

After all cuts, the background is dominated by $K_{L} \rightarrow \pi^{0} \pi^{0} \pi^{0}$ events, with the total background constituting approximately $30 \%$ of the final events. The main sources of systematic uncertainty stem from understanding the $3 \pi^{0}$ background and its normalization. Other sources of systematic uncertainty come from our knowledge of the photon veto system, the acceptance determination and external factors such as the measured $K_{L} \rightarrow \pi^{0} \pi^{0}$ branching ratios. The total systematic uncertainty is $2.9 \%$.

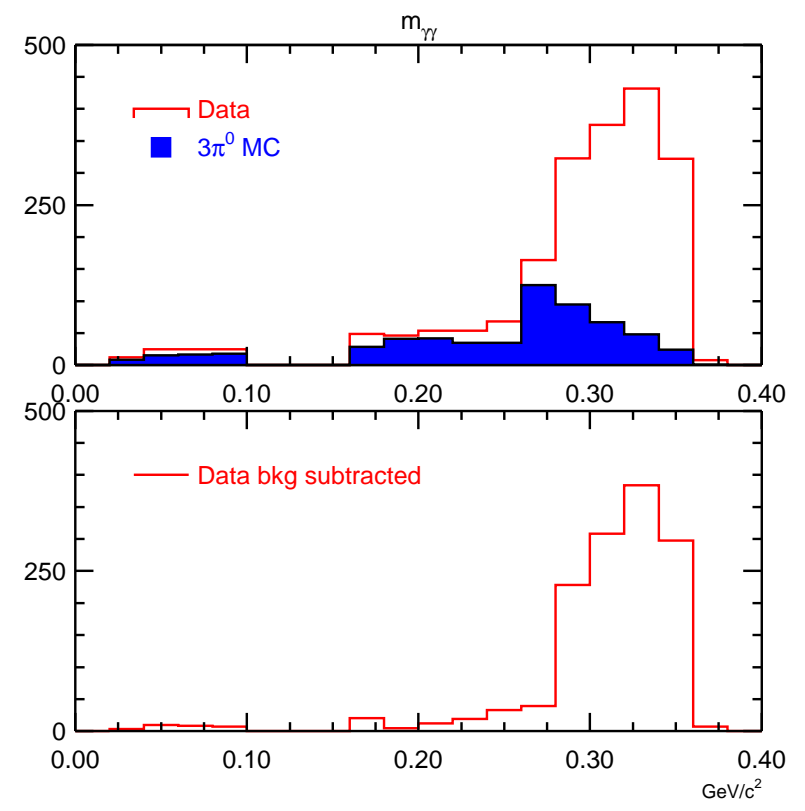

Figure 1: The $\gamma \gamma$ mass distribution for $K_{L} \rightarrow \pi^{0} \gamma \gamma$ candidates before (top) and after (bottom) background subtraction.

After all cuts have been implemented, we find 1982 events with a background of 601 events. 
The reconstructed $\gamma \gamma$ mass is shown in Figure 1. The distinctive $\gamma \gamma$ shape results from coupling of the $\gamma \gamma$ system to two virtual pions, and peaks around $320 \mathrm{MeV} / c^{2}$. Using the decay $K_{L} \rightarrow$ $\pi^{0} \pi^{0}$ as normalization, we determine the $K_{L} \rightarrow \pi^{0} \gamma \gamma$ branching ratio to be: $\operatorname{BR}\left(K_{L} \rightarrow \pi^{0} \gamma \gamma\right)=$ $(1.30 \pm 0.03 \pm 0.04) \times 10^{-6}$, where the first error is statistical and the second error systematic. The error from the background events is included in our systematic error. This result is compatible with the result from NA48 [8]. Our new result supercedes the previous $\mathrm{KTeV}$ measurement of $\mathrm{BR}\left(K_{L} \rightarrow \pi^{0} \gamma \gamma\right)[9]$.

\section{Measurement of $K_{L} \rightarrow \pi^{0} e^{+} e^{-} \gamma$}

The decay $K_{L} \rightarrow \pi^{0} e^{+} e^{-} \gamma$ produces two charged tracks in the spectrometer and three photons in the CsI calorimeter. The three photons can be combined in three different ways to form a neutral pion. We choose the combination that has the best $\pi^{0}$ mass. The neutral vertex is used to determine the decay position rather than the charged vertex due to its better resolution.

Like the $K_{L} \rightarrow \pi^{0} \gamma \gamma$ decay, the main backgrounds to this decay are from $K_{L} \rightarrow \pi^{0} \pi^{0}$ and $K_{L} \rightarrow \pi^{0} \pi^{0} \pi^{0}$. The difference is that one of the $\pi^{0}$ undergoes Dalitz decay to $e^{+} e^{-} \gamma$. To help reduce the background from $K_{L} \rightarrow \pi^{0} \pi^{0}$ decays, we formed a neural net variable using the reconstructed masses $m_{\gamma \gamma}$ and $m_{e^{+} e^{-} \gamma}$ from the second and third best combinations. We define the second and third best combinations using the difference between the $\gamma \gamma$ invariant mass and the $\pi^{0}$ mass.

To reduce $K_{L} \rightarrow \pi^{0} \pi^{0} \pi^{0}$ backgrounds we also require the photon shape variable, defined above, to be small for each photon candidate. We further reduce this background by defining a variable $p_{L}^{2}$, which is the longitudinal momentum squared of the missing $\pi^{0}$ in the kaon rest frame. We perform a two-dimensional cut on the $p_{L}^{2}$ variable versus the three photon invariant mass, $m_{\gamma \gamma \gamma}$.

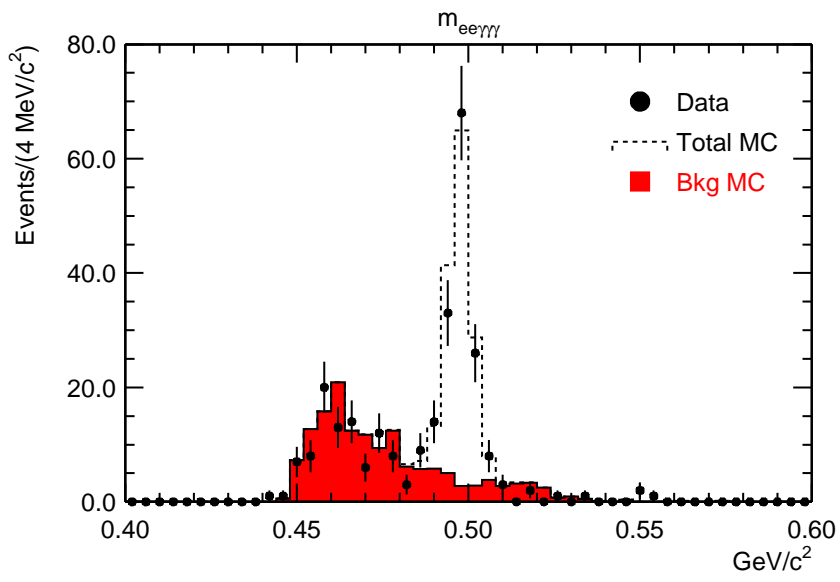

Figure 2: The $e^{+} e^{-} \gamma \gamma \gamma$ mass for the combined 1997 and 1999 data sets. The dots are the data, the red histogram is the sum of the background Monte Carlo, and the dashed histogram represents the sum of the $K_{L} \rightarrow \pi^{0} \pi^{0}$ and $K_{L} \rightarrow \pi^{0} \pi^{0} \pi^{0}$ backgrounds.

After applying all cuts, we find 139 events over a background of 14.4 events as shown in Figure 2. We reconstructed $80,445 K_{L} \rightarrow \pi^{0} \pi^{0}$ events used for normalization. This allows us 
to determine the branching ratio for $K_{L} \rightarrow \pi^{0} e^{+} e^{-} \gamma$ to be $\mathrm{BR}\left(K_{L} \rightarrow \pi^{0} e^{+} e^{-} \gamma\right)=(1.90 \pm 0.16 \pm$ $0.12) \times 10^{-8}$ where the first error is statistical and the second is systematic. As in the $K_{L} \rightarrow \pi^{0} \gamma \gamma$, the normalization mode branching ratio has decreased by approximately $8 \%$ from the value used in the published $\mathrm{KTeV}$ result. The total systematic uncertainty associated with the branching ratio measurement is $6.4 \%$. The largest systematic comes from the limited Monte Carlo statistics.

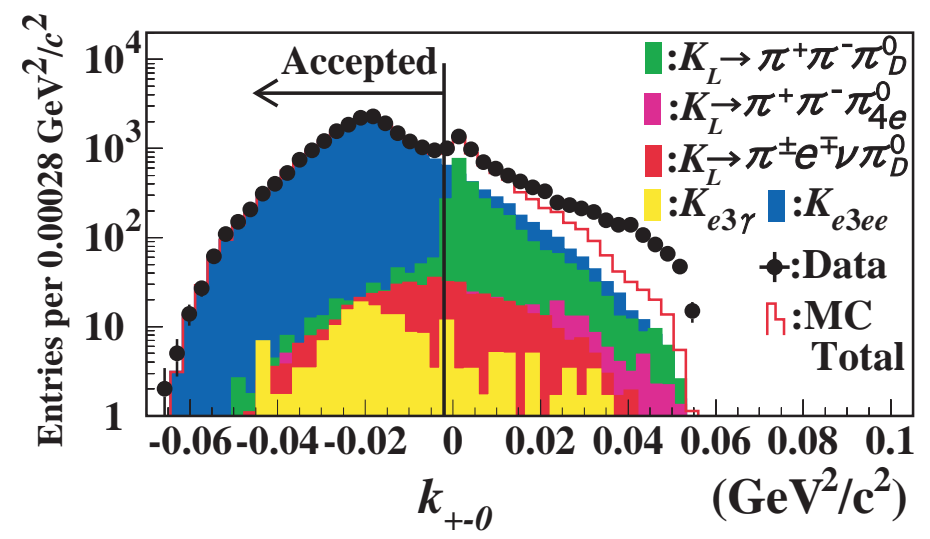

Figure 3: The $k_{+-0}$ variable as defined in the text. The blue histogram represents the $K_{L} \rightarrow \pi^{ \pm} e^{\mp} v e^{+} e^{-}$ signal, while the other histograms represent the various kaon backgrounds.

\section{Measurement of $K_{L} \rightarrow \pi^{ \pm} e^{\mp} v e^{+} e^{-}$}

Until now the decay $K_{L} \rightarrow \pi^{ \pm} e^{\mp} v e^{+} e^{-}$has not been seen. The final state consists of four tracks: three electrons and a pion. Of the two possible $e^{+} e^{-}$combinations, we choose the one with the smallest invariant mass.

The most significant backgrounds result from the following decays: $K_{L} \rightarrow \pi^{+} \pi^{-} \pi_{D}^{0}, K_{L} \rightarrow$ $\pi^{ \pm} e^{\mp} v \pi_{D}^{0}, K_{L} \rightarrow \pi^{+} \pi^{-} \pi_{4 e}^{0}$, and $K_{L} \rightarrow \pi^{ \pm} e^{\mp} v \gamma$, where $\pi_{D}^{0}$ is $\pi^{0} \rightarrow e^{+} e^{-} \gamma$ and $\pi_{4 e}^{0}$ is $\pi^{0} \rightarrow e^{+} e^{-} e^{+} e^{-}$. Backgrounds involving misidentified pions are reduced through particle identification. We require the ratio of the energy measured in the calorimeter to the momenta measured in the spectrometer $(E / p)$ to be close to one. Also, we require a good electron probability in the TRD system. As can be seen in Fig. 3, the largest background is $K_{L} \rightarrow \pi^{+} \pi^{-} \pi_{D}^{0}$. One can significantly reduce this background by defining a variable $k_{+-0}$, which is the longitudinal momentum squared of the $\pi^{0}$ in the frame where the $\pi^{+} \pi^{-}$is transverse to the $K_{L}$. For $K_{L} \rightarrow \pi^{+} \pi^{-} \pi_{D}^{0}$ events this variable will be positive, while for a significant fraction of the $K_{L} \rightarrow \pi^{ \pm} e^{\mp} v e^{+} e^{-}$events this variable is negative.

Applying the cut shown in Fig. 3, we find 20,225 candidate events over a background of $1017.1 \pm 24.7$ events. The largest systematics associated with the branching ratio measurement are the unobserved photon in the normalization mode, the event selection criteria and the radiative corrections. Using the $K_{L} \rightarrow \pi^{+} \pi^{-} \pi_{D}^{0}$ events as normalization, we determine the branching ratio to be $\operatorname{BR}\left(K_{L} \rightarrow \pi^{ \pm} e^{\mp} v e^{+} e^{-}\right)=(1.29 \pm 0.01 \pm 0.04) \times 10^{-5}$ with $M_{e^{+} e^{-}}>5 \mathrm{MeV} / c^{2}, E_{e^{+} e^{-}}^{*}>30$ $\mathrm{MeV}$. This is the first measurement of this mode and is consistent with $\operatorname{NLO}\left(p^{4}\right)$ chiral perturbation theory. Comparisons between our data and $\mathrm{LO}\left(p^{4}\right)$ chiral perturbation calculations disfavor these theoretical predictions. 


\section{Summary and Conclusions}

The $\mathrm{KTeV}$ experiment has presented three new results on $K_{L} \rightarrow \pi^{0} \gamma \gamma, K_{L} \rightarrow \pi^{0} e^{+} e^{-} \gamma$ and $K_{L} \rightarrow \pi^{ \pm} e^{\mp} v e^{+} e^{-}$. The first result is competitive with the world's best result from NA48, while the other two represent the world's best measurements on these two decays. The measured branching ratios are all consistent with the most recent chiral perturbation calculations.

\section{References}

[1] G.D. Barr et al., Phys. Lett. B284, 440 (1992).

[2] V. Papadimitriou et al. Phys. Rev. D44, 573 (1991).

[3] G. D’Ambrosio and J. Portoles, Nucl. Phys. B492, 417 (1997).

[4] J. Donoghue and F. Gabbiani, Phys. Rev. D56, 1605 (1997).

[5] J. Gasser et al., Eur. Phys. J. C40 205 (2005).

[6] J.R. Batley et al., Phys. Lett. B576, 43 (2003).

[7] J.R. Batley et al., Phys. Lett. B599, 197 (2004)..

[8] A. Lai et al., Phys. Lett. B536, 229 (2002).

[9] A. Alavi-Harati et al., Phys. Rev. Lett. 83, 917 (1999).

[10] G. D’Ambrosio, G. Ecker, G. Isidori and J. Portoles, J. High Energy Physics, 08, 004 (1998). 